\title{
EVALUACIÓN DE LA SATISFACCIÓN DE ESTUDIANTES UNIVERSITARIOS DE NUEVO INGRESO CON EL PROGRAMA E.S.C.L.O.S.A.: EXPERIENCIA DE UN PROGRAMA DE ORIENTACIÓN
}

\section{SATISFACTION ASSESSMENT OF FRESHMEN UNIVERSITY BY "ESCLOSA" PROGRAMME: A GUIDANCE PROGRAMME EXPERIENCE}

\author{
Carmen María Hernández Garre ${ }^{1}$ \\ Universidad de Almería. Facultad de Ciencias de la Educación \\ Almería, España \\ María Dolores López Justicia \\ Eva María Olmedo Moreno \\ Universidad de Granada. Facultad de Ciencias de la Educación \\ Granada, España
}

\section{RESUMEN}

La adaptación académica de estudiantes noveles al ámbito universitario supone un proceso complejo en el que se demandan exigencias a todos los niveles, siendo necesaria una orientación en Educación Superior que garantice la atención en los diferentes aspectos, tanto académicos como sociopersonales de los estudiantes. En el artículo se presenta, de forma sintética la descripción e implementación así como la evaluación de un programa de orientación e intervención psicopedagógica para facilitar la adaptación de estudiantes universitarios noveles. El objetivo fundamental de la actividad fue formar, orientar y capacitar sobre estrategias académicas (lectura comprensiva, ortografía, hábitos, técnicas de estudio y autorregulación) y sociopersonales (habilidades sociales) a un grupo de estudiantes universitarios de la titulación de Maestro en Educación Infantil, matriculados en la Facultad de Ciencias de la Educación de la Universidad de Granada. Habiéndose analizado previamente las necesidades relacionadas con dichas variables las cuales fueron publicadas en los estudios de López-Justicia, Hernández, Jiménez, Polo y

Correspondencia: Carmen María Hernández Garre: Carretera de Sacramento s/n 04120. Almería. Correo-e: cmhgarre@ual.es 
Chacón (2008) y López-Justicia, Hernández, Jiménez, Polo, Chacón y González (2008). programa se organizó en diferentes talleres con una estructura teórico-práctica.

Los resultados obtenidos de la valoración del programa permiten concluir que la iniciativa llevada a cabo ha sido de interés y utilidad para los participantes, al haber destacado la importancia de la actividad para su formación, así como el interés por los temas propuestos.

Palabras clave: Alumnado universitario, Orientación, Adaptación académica, Éxito académico, Abandono.

\section{ABSTRACT}

Academic adjustment of novel students in university level involve a complex process with demanded requirements on several scenes, being needed a High Education Guidance that ensure attention to different aspects both academic and socio personal of students. Realizing there these potential needs at the beginning of his academic career. Should be a priority for universities, because they are linked to the student's academic performance and their retention at institutions. This paper shows a summarized development of a guidance programme and psycho-pedagogical intervention to facilitate the initial adjustment of these students. The main aim was related to train, guide and qualify about academic skills (reading comprehension, spelling, habits, study techniques and self-regulation) and socio-personal or social skills to students group of Bachelor to become Kindergarten Teachers, enrolled in the Faculty of Education at University of Granada. Having analyzed previously needs related to all those variables published in studies by López-Justicia, Hernandez , Jiménez , Polo y Chacón (2008) and López- Justicia , Hernández , Jiménez , Polo, Chacón y González ( 2008). The programme was organized around different workshops from a theoretical and practical structure. The assessment outcomes shows that activities have been interesting and useful for users, having stressed its importance for their training and refreshing of topics proposed.

Key Words: University students, Guidance, Academic adjustment, Academic success, Dropout.

\section{Introducción}

El comienzo de la etapa formativa universitaria es un periodo complejo para la adaptación de los estudiantes a esta nueva realidad, ya que comporta cambios personales, académicos y sociales en el estudiante (Rué, 2014). A nivel académico, requiere que el estudiante asuma un mayor nivel de exigencia en contenidos formativos, mediante un enfoque de mayor autonomía. A nivel socioafectivo, son necesarias unas adecuadas destrezas sociales para su desenvolvimiento en el trabajo grupal y la convivencia para mejorar la vida del estudiante.

El éxito académico de los estudiantes se ve condicionado sobre todo, por una adecuada adaptación a los estudios y a la vida en la universidad, más aún cuando la tasa de abandono del sistema universitario español que se produce en los primeros años, es elevada (Michavila, García, Martínez, Merhi, Esteve, Martínez, 2012; Olmedo, 2013), ya que, según las estadísticas, como mínimo uno de cada cuatro alumnos de nuevo ingreso abandonará sus estudios superiores (Rué, 2014). De ahí la importancia de las acciones de integración dirigidas al alumnado universitario novel que faciliten su transición a la nueva etapa. 
Actualmente, la Educación Superior entendida como promovedora del desarrollo integral del estudiante, demanda una orientación que favorezca el desarrollo personal, educativo y profesional del alumnado (Álvarez, Pérez-Jorge, López y González, 2014) tal y como demandan los planteamientos a nivel europeo, atendiendo a las necesidades que han ido manifestándose en este nivel educativo.

Aunque, en general, las acciones de orientación que se están llevando a cabo en las instituciones de educación superior, giran en torno a 4 momentos de orientación (Gairín, Muñoz, Galán-Mañas, Fernández y Sanahuja, 2014): Antes del ingreso en la universidad, la inserción en la vida universitaria, el desarrollo del perfil (durante la vida universitaria) y la transición al término del grado, siguen siendo acciones con un carácter puntual; ya que ofertan actividades dentro de cada uno de estos momentos, de forma aislada (Grau, Álvarez, Moncho, Ramos, Crespo y Alonso, 2013; Sanz, 2011). Es por ello, que cada vez más, se demandan acciones orientadoras dirigidas hacia el desarrollo personal y profesional del estudiante (habilidades sociales, autoconcepto, autorregulación, etc.) de una manera holística (García, Conejero y Díez, 2014). Desde esta perspectiva, nos planteamos la realización de un programa de entrenamiento para desarrollar habilidades académicas de lectura, hábitos de estudio, autorregulación y habilidades sociales para la adaptación a los estudios universitarios; siendo el objetivo principal de la actividad informar y dotar a través de una acción práctica (a modo de talleres), a un grupo de estudiantes universitarios de nuevo ingreso de la Facultad de Ciencias de la Educación de la UGR. Se trataba de reforzar en ellos los conocimientos y destrezas necesarias para desarrollar su actividad académica acorde con las exigencias universitarias actuales.

En este trabajo se pretendía además, conocer la satisfacción del alumnado con respecto al programa de orientación E.S.C.L.O.S.A., en el que participaron, y cuyo modelo de evaluación de programas es CIPP de Stufflebeam (1996).

\section{Método}

\section{Muestra}

La selección de la muestra obedece a un muestreo aleatorio simple entre los estudiantes de primero de la titulación de Grado de Maestro en Educación Infantil. Se selecciona a una muestra de 50 de los que finalmente participan 40. Estos a su vez se dividieron de forma aleatoria en 20 estudiantes para el grupo control y 20 estudiantes para el grupo experimental. Por lo que la evaluación de la satisfacción del alumnado, con respecto al programa E.S.C.L.O.S.A., se realizó con el grupo experimental. De tal modo que el programa se implementó en 20 estudiantes pertenecientes a dicha titulación, con edades comprendidas entre los 18 y 20 años.

\section{Procedimiento: Descripción del programa E.S.C.L.O.S.A.}

Para la realización del presente estudio, en primer lugar se convocó a los estudiantes de la titulación a una reunión informativa en enero del curso 2009/2010, donde se les informó de la actividad y de la participación voluntaria en la misma.

Una vez recogidos los datos de los participantes en el estudio, se les convocó vía telefónica informándoles sobre los objetivos y finalidad, así como la fecha de inicio y el horario de los talleres del programa orientación e intervención psicopedagógica E.S.C.L.O.S.A. 
El programa se desarrolló a través de diferentes talleres formativos que incluían un número de sesiones presenciales (32 horas) y sesiones no presenciales (16 horas) centradas en asesoramiento y entrenamiento en las áreas deficitarias detectadas en el análisis de necesidades de los estudiantes de dicha titulación, recogido en estudios previos (López-Justicia, Hernández, Fernández, Polo y Chacón, 2008; López-Justicia, Hernández, Polo, Fernández, Chacón y González, 2008), tales como; lectura comprensiva y ortografía, la mejora de hábitos y técnicas de estudio, entrenamiento en habilidades sociales y estrategias de autorregulación.

Los diferentes talleres, se desarrollaron a lo largo de 8 semanas, con una duración de 4 horas semanales, en horario de mañana o tarde quedando organizado del siguiente modo:

Taller de Comprensión Lectora. Este taller se desarrolló durante 4 sesiones en las que se ofrecieron diversas estrategias para la mejora de la comprensión lectora (CL en adelante) y donde los estudiantes pudieron poner en práctica las técnicas más eficaces para una lectura comprensiva (ver Tabla 1).

La lectura constituye una de las actividades más frecuentes y demandadas en el ámbito universitario, por tanto, comprender el significado de lo que se lee se convierte en un aspecto esencial a todos los niveles, siendo la herramienta principal de aprendizaje para los estudiantes en el ámbito de la educación superior. De ahí la importancia de incluir estos contenidos en el programa de orientación.

TABLA 1. Taller de Comprensión Lectora

\begin{tabular}{cllc}
\hline & \multicolumn{1}{c}{ CONTENIDOS } & \multicolumn{1}{c}{ OBJETIVOS } & DURACIÓN \\
\hline Sesión 1 & $\begin{array}{l}\text { La comprensión lectora. La } \\
\text { eficacia lectora }\end{array}$ & $\begin{array}{l}\text { Tomar conciencia de los } \\
\text { distintos procesos implicados } \\
\text { en la lectura }\end{array}$ & 2 horas \\
Sesión 2 & $\begin{array}{l}\text { Técnicas para la comprensión. } \\
\text { El resumen y el subrayado }\end{array}$ & $\begin{array}{l}\text { Conocer y saber utilizar las } \\
\text { estrategias más eficaces para } \\
\text { facilitar la CL. }\end{array}$ & 2 horas \\
Sesión 3 & $\begin{array}{l}\text { Técnicas para la comprensión. } \\
\text { El esquema y el mapa } \\
\text { conceptual }\end{array}$ & $\begin{array}{l}\text { Conocer y saber utilizar las } \\
\text { estrategias más eficaces para } \\
\text { facilitar la CL }\end{array}$ & 2 horas \\
Sesión 4 & $\begin{array}{l}\text { La composición de textos: la } \\
\text { redacción }\end{array}$ & $\begin{array}{l}\text { Saber el procedimiento } \\
\text { correcto en la elaboración de } \\
\text { textos. }\end{array}$ & 2 horas \\
\hline
\end{tabular}

Taller de Ortografía. Para llevar a cabo este taller, los estudiantes completaron ejercicios de escritura y ortografía siguiendo un cuadernillo (Pérez, Cañado y Pérez, 2000) mediante el cual, tomaron conciencia de sus errores ortográficos y practicaron las normas correctas de escritura (ver Tabla 2). 
TABLA 2. Taller de Ortografía

\begin{tabular}{llll}
\hline & \multicolumn{1}{c}{ CONTENIDOS } & \multicolumn{1}{c}{ OBJETIVOS } & DURACIÓN \\
\hline Sesión 1 & Ortografía. Normas básicas. & $\begin{array}{l}\text { Tomar conciencia de las distintas } \\
\text { normas ortográficas. }\end{array}$ & 2 horas \\
Sesión 2 & Vocabulario básico. & $\begin{array}{l}\text { Conocer y saber utilizar las } \\
\text { normas básicas de ortografía. }\end{array}$ & 2 horas \\
Sesión 3 & $\begin{array}{l}\text { Acentuación de formas verbales } \\
\text { con pronombres enclíticos. }\end{array}$ & $\begin{array}{l}\text { Conocer las reglas ortográficas de } \\
\text { determinadas formas verbales. } \\
\text { Saber situar los signos de } \\
\text { suntuación en un texto. }\end{array}$ & 2 horas \\
Sesión 4 & Signos de puntuación. & 2 horas \\
\hline
\end{tabular}

Taller de Hábitos, Técnicas de Estudio y Autorregulación. En este taller se abordaron de forma práctica diferentes aspectos relacionados con el estudio y la gestión de las tareas académicas. Así, en la primera sesión los alumnos tomaron conciencia sobre sus hábitos y estrategias utilizadas. Una vez identificadas las situaciones de riesgo para el estudio, es preciso reflexionar sobre la importancia de la alimentación y el sueño en su vida académica, así como conocer diferentes técnicas de relajación para afrontar el estudio.

En las siguientes y últimas sesiones, practicaron ejercicios de autorregulación acerca de la planificación de las tareas académicas, la gestión del tiempo empleado y la importancia de tomar buenos apuntes (ver Tabla 3).

Mediante un adecuado entrenamiento en estas dimensiones, los estudiantes mejoran su grado de control sobre el aprendizaje y el rendimiento, paliando muchas de las dificultades de aprendizaje académicas, evitando, por ejemplo, conductas de procastinación (ver Figura 1).

TABLA 3. Taller de Hábitos, Técnicas de Estudio y Autorregulación

\begin{tabular}{|c|c|c|c|}
\hline & CONTENIDOS & OBJETIVOS & DURACIÓN \\
\hline Sesión 1 & $\begin{array}{l}\text { Presentación, toma de contacto } \\
\text { y revisión de metas }\end{array}$ & $\begin{array}{l}\text { Revisar metas y objetivos personales con } \\
\text { respecto a los estudios. } \\
\text { Generar toma de conciencia relacionada con } \\
\text { buenos y malos hábitos para el estudio. } \\
\text { Identificar situaciones de riesgo para el estudio. } \\
\text { Ayudar a los alumnos a tomar conciencia sobre } \\
\text { las técnicas y estrategias de estudio que } \\
\text { utilizan. }\end{array}$ & 2 horas \\
\hline Sesión 2 & $\begin{array}{l}\text { Necesidades básicas } \\
\text { relacionadas con el estudio y } \\
\text { formas de afrontar diferentes } \\
\text { modelos de examen }\end{array}$ & $\begin{array}{l}\text { Tomar conciencia de la importancia de los } \\
\text { hábitos alimenticios para su vida académica e } \\
\text { importancia del sueño. } \\
\text { Pensar en la importancia de la actividad física y } \\
\text { el deporte para los estudios. } \\
\text { Reflexionar sobre el estudio para los diferentes } \\
\text { tipos de exámenes. } \\
\text { Conocer diferentes técnicas de relajación. }\end{array}$ & 2 horas \\
\hline Sesión 3 & $\begin{array}{l}\text { Autorregulación y planificación } \\
\text { de tareas }\end{array}$ & $\begin{array}{l}\text { Ayudar a los alumnos a que tomen conciencia } \\
\text { de la importancia de planificar las tareas } \\
\text { académicas. }\end{array}$ & 2 horas \\
\hline
\end{tabular}




\begin{tabular}{|c|c|c|c|}
\hline Sesión 4 & $\begin{array}{l}\text { Autorregulación y toma de } \\
\text { apuntes }\end{array}$ & $\begin{array}{l}\text { Tomar conciencia del tiempo empleado en } \\
\text { actividades cotidianas para poder tomar } \\
\text { decisiones al respecto. } \\
\text { Reflexionar sobre la importancia de tomar } \\
\text { buenos apuntes. }\end{array}$ & 2 horas \\
\hline
\end{tabular}

FIGURA 1. Sesión 4 - Autorregulación

Taller de Hábitos, Técnicas de estudio y Autorregulación

SESIÓN 4 Autorregulación

Acfividad 7: Identifica y anota los 5 argumentos más frecuentes para procrastinar (aplazar) el estudio personal y la realización de las tareas acodémicas.

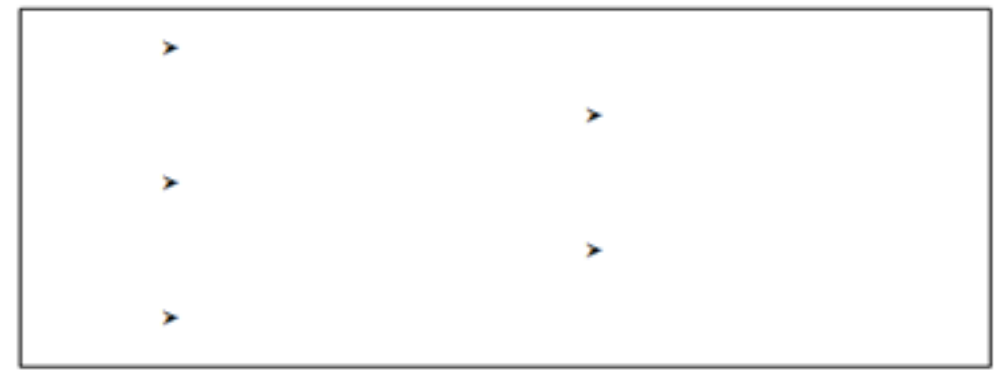

Actividad 8: Identifica los 5 principales distractores internos y externos que perturban tu aprendizaje y tu estudio y sugiere formas de evitarlos para poder concentrorte en la torea.

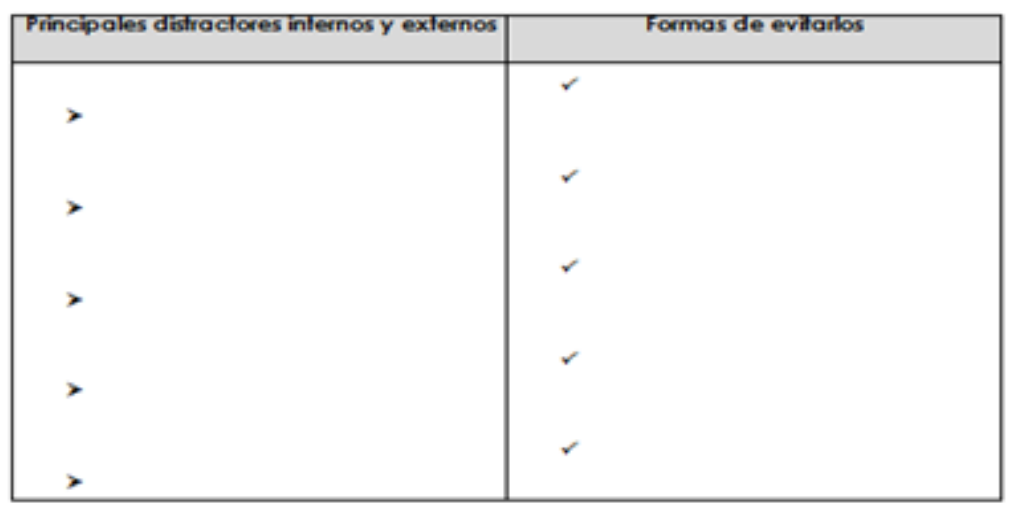

Taller de Habilidades Sociales. Los estudiantes participantes realizaron un entrenamiento en distintas dimensiones de las habilidades sociales, tales como la expresión adecuada de molestia o desagrado, defender sus derechos personales (ver Figura 2), o hablar en público, entre otras (ver Tabla 4).

Las habilidades sociales constituyen una herramienta de gran utilidad a lo largo de la vida para interrelacionarse entre sí y lograr cubrir nuestras necesidades. El alumnado universitario en concreto, se desenvuelve en un entorno diario con demandas sociales crecientes y que generan gran cantidad de estresores, tanto a nivel personal como los relacionados con su actividad como estudiantes. Es evidente que la educación superior no puede ser ajena a esta demanda, de ahí que se contemple en el presente programa. 
TABLA 4. Taller de Habilidades Sociales

\begin{tabular}{|c|c|c|c|}
\hline & CONTENIDOS & OBJETIVOS & DURACIÓN \\
\hline Sesión 1 & $\begin{array}{l}\text { Presentación y toma de } \\
\text { contacto. ¿Para qué las } \\
\text { habilidades sociales? }\end{array}$ & $\begin{array}{l}\text { - Fomentar el conocimiento entre los } \\
\text { miembros del grupo y el monitor/a. } \\
\text { - Aclaración de términos e importancia de las } \\
\text { Habilidades Interpersonales. } \\
\text { - Identificar los estilos de respuesta sumiso, } \\
\text { asertivo y agresivo. }\end{array}$ & 1 hora \\
\hline Sesión 2 & $\begin{array}{l}\text { Saber expresar molestia, } \\
\text { desagrado y/o disgusto } \\
\text { adecuadamente }\end{array}$ & $\begin{array}{l}\text { - Aclarar y aprender lo que significa expresar } \\
\text { molestia, desagrado y/o disgusto. } \\
\text { - Conocer las ventajas y desventajas de dicha } \\
\text { habilidad social. } \\
\text { - } \\
\text { Representar diferentes situaciones por parte } \\
\text { de los alumnos sobre dicha habilidad. } \\
\text { - Adquirir y mejorar las capacidades de } \\
\text { expresar molestia, desagrado y/o disgusto. }\end{array}$ & \\
\hline Sesión 3 & $\begin{array}{l}\text { ¿Cómo defender tus } \\
\text { derechos personales? }\end{array}$ & $\begin{array}{l}\text { - Conocer qué y cuáles son los derechos } \\
\text { personales. } \\
\text { - Conocer las ventajas y desventajas de } \\
\text { hacerlo o no convenientemente. } \\
\text { - Representar diferentes situaciones por parte } \\
\text { de los alumnos de dicha habilidad. } \\
\text { - Defender adecuada y eficazmente los } \\
\text { derechos personales. }\end{array}$ & 1 hora \\
\hline Sesión 4 & Decir "No" & $\begin{array}{l}\text { - Saber en qué consiste decir "no" o rechazar } \\
\text { una petición y conocer sus ventajas e } \\
\text { inconvenientes. } \\
\text { - Representar diferentes situaciones donde se } \\
\text { ponga de manifiesto dicha habilidad. } \\
\text { - Adquirir/mejorar la capacidad de decir "no" o } \\
\text { rechazar una petición. }\end{array}$ & 1 hora \\
\hline Sesión 5 & $\begin{array}{l}\text { Saber hacer críticas y } \\
\text { recibir críticas }\end{array}$ & $\begin{array}{l}\text { - } \quad \text { Conocer en qué consiste realizar críticas. } \\
\text { - } \text { aprender esta habilidad. } \\
\text { - Proporcionar pautas para aprender a recibir } \\
\text { críticas y responder ante una queja. }\end{array}$ & 1 hora \\
\hline Sesión 6 & La comunicación no verbal & $\begin{array}{l}\text { - Reconocer la importancia de la comunicación } \\
\text { no verbal. } \\
\text { - Saber los sistemas de codificación incluidos } \\
\text { en este lenguaje y su significado. } \\
\text { - Facilitar el proceso de comunicación } \\
\text { interpersonal. }\end{array}$ & 1 hora \\
\hline Sesión 7 & Hablar en público & $\begin{array}{l}\text { - Tomar conciencia de la importancia que } \\
\text { supone hablar en público sin problemas. } \\
\text { - Poner en práctica la habilidad. }\end{array}$ & 1 hora \\
\hline Sesión 8 & $\begin{array}{l}\text { Repaso de todas las } \\
\text { habilidades interpersonales }\end{array}$ & $\begin{array}{l}\text { - Poner en práctica las habilidades } \\
\text { interpersonales más importantes. }\end{array}$ & 1 hora \\
\hline
\end{tabular}


FIGURA 2. Sesión 3 - ¿Cómo defender tus derechos personales?

SESIÓN 3 ¿Cómo puedes defender tus derechos personales?

Actividad 4 Registro de situaciones. Identificación de estilos de respuesta + Diapositiva con las situaciones.

\begin{tabular}{|c|c|c|c|}
\hline & Pasivo / sumiso & $\begin{array}{c}\text { Habilidoso / } \\
\text { Asertivo }\end{array}$ & Agresivo \\
\hline \multicolumn{4}{|c|}{ Sifuacion $n^{\circ}$ 1: Estos en la barra de una cafeteria... } \\
\hline \multicolumn{4}{|l|}{ Escena $n^{\circ} 1$} \\
\hline \multicolumn{4}{|l|}{ Escena $n^{\circ} 2$} \\
\hline \multicolumn{4}{|l|}{ Escena $n^{\circ} 3$} \\
\hline \multicolumn{4}{|c|}{ Sifudción $n^{\circ}$ 2: Estas en una hamburgueseria... } \\
\hline \multicolumn{4}{|l|}{ Escena $n^{\circ} 1$} \\
\hline \multicolumn{4}{|l|}{ Escena $n^{\circ} 2$} \\
\hline \multicolumn{4}{|l|}{ Escena $n^{\circ} 3$} \\
\hline \multicolumn{4}{|c|}{ Sifuación $n^{\circ} 3$ : vas a una tienda a comprar un CD... } \\
\hline \multicolumn{4}{|l|}{ Escena $n^{\circ} 1$} \\
\hline \multicolumn{4}{|l|}{ Escena $n^{\circ} 2$} \\
\hline Escena $n^{\circ} 3$ & & & \\
\hline
\end{tabular}

Pasos necesarios para defender los derechos personales adecuadamente - Conocer fus derechos.

Encontrar elmomento y lugar mós idóneos (cuando esté tranquilo, a solos...].

3. Transmitir un mensoje respetucsoy educodo, directo, sin rodeos, breve $y$ claro.

4. Utilizor el pronombre "yo" ("yo creo.... yo siento, yo pienso...).

5. Acercarse a la otra persona, utilizar un tono de voz firme y tranquila, no hablar ni muy despocio ni muy rápido, mirar a los ojos de la otra persona, pero sin fileza y adoptor una postura corporal natural.

6. Comunicar a la otra persona que nos está tratando injustamente, que no queremos nocer algo que nos pide o que estánociendo algo que nos periudica.

7. Agrodecer a la otra persona el hoberte escuchodo.

Las sesiones no presenciales consistieron en la realización, por parte del alumnado, de actividades relacionadas con los contenidos del programa para que pudieran poner en práctica los conocimientos adquiridos. Estas sesiones incluían actividades para la mejora de la comprensión lectora a través de la lectura de textos, ejercicios de escritura y ortografía, habilidades sociales y ejercicios sobre técnicas y hábitos de estudio.

Una vez concluido este proceso, se concreta un día para su entrega y posteriormente se les cita individualmente mediante tutorías presenciales para proporcionarles feedback sobre sus ejercicios y corregir los posibles errores. La duración total de estas sesiones fue de 16 horas.

\section{Instrumentos}

Al finalizar la implementación del programa se llevó a cabo una evaluación de la satisfacción del estudiante con respecto al programa, a través de un cuestionario de valoración de dicha actividad, que incluía una valoración en diferentes aspectos: A. Organización del curso, B. Motivo de asistencia, C. Expectativas, D. Contenidos y metodología, E. Duración, F. Docentes, G. Equipamiento y medios técnicos, $\mathrm{H}$. Espacios, instalaciones y mobiliario e I. Comentarios y sugerencias. $1-5$.

El cuestionario se compone de 26 ítems mediante una escala de tipo Likert que oscilan entre 
Después de haber confeccionado dicho cuestionario provisionalmente, se realizó la validez de contenido del mismo, mediante jueces expertos. Para ello se contó con dos expertos en metodología de la investigación educativa y dos expertos en psicología evolutiva y de la educación. A todos se les solicitó colaboración en la realización de una valoración personal del cuestionario globalmente, y de los ítems que lo componen, en cuanto a:

1. En qué grado los ítems subdivididos y presentados por bloques, representan dichas dimensiones.

2. En qué medida cada uno de los ítems mide la satisfacción del alumnado con el programa E.S.C.L.O.S.A.

3. El grado de claridad e inteligibilidad en la formulación de los ítems.

Una vez realizado el análisis por cada experto, se calculó el grado de acuerdo para garantizar la objetividad y la fiabilidad interjueces, mediante el índice de acuerdo entre observadores denominado índice de porcentaje de concordancia ínter expertos ([ $n^{\circ}$ de acuerdos / $\left(n^{\circ}\right.$ de acuerdos $+n^{\circ}$ de desacuerdos) $\times 100$ ). En este estudio se han obtenido, en la mayoría de los ítems y dimensiones, valores para este coeficiente de $\mathrm{K}=0.76$, lo que refleja un acuerdo excelente.

Con esta perspectiva de acuerdo, se pasó a modificar y depurar el cuestionario en base a las orientaciones aportadas por los jueces, quedando finalmente conformado en las dimensiones e ítems que se presenta en la Tabla 5.

\section{Resultados}

A continuación se presentan los resultados de la valoración que los estudiantes participantes hicieron del programa desarrollado en cuatro talleres. El cuestionario de valoración del programa fue validado por jueces expertos. Los resultados obtenidos se agrupan en los nueve apartados señalados:

- Organización del curso: Un alto porcentaje del alumnado estaba totalmente de acuerdo con la información que se le ha proporcionado, el cumplimiento de fechas y horarios, y con el material proporcionado.

- Motivo de asistencia: Cuando se les preguntó por las razones que les llevaron a asistir a los talleres que conformaban el programa de intervención, la mayoría afirma que es necesario para su formación, decide realizarlos por poder obtener créditos de Libre Configuración y muchos de ellos lo decide realizar por el interés por los temas propuestos.

- Expectativas: Es importante señalar que casi la totalidad de los estudiantes participantes están de acuerdo con que el programa ha respondido a las expectativas que tenían sobre éste.

- Contenidos y Metodología: La información relativa a los contenidos para el logro de los objetivos de los talleres, los contenidos para la formación académica y personal, la combinación entre teoría y práctica, los ejercicios y trabajos prácticos y el uso de recursos didácticos y demás material utilizado, en general se consideraron adecuados y abundantes. 
- Duración: En cuanto a la duración del programa, la mayoría opinó que fue suficiente para abordar los contenidos, así como con el horario, ya que lo consideraron favorecedor para la asistencia a los talleres que conformaban el programa.

- Docentes: A nivel personal, en un rango de 1-5, los alumnos destacaron la capacidad para solventar dudas y la claridad expositiva para la realización de las tareas.

- Equipamiento y medios técnicos: En cuanto al equipamiento y medios técnicos para el desarrollo de la actividad, la mayoría los consideraron adecuados y suficientes para el desarrollo del contenido del curso.

- Espacios, instalaciones y mobiliario: En líneas generales, los participantes consideraron adecuados las prestaciones que se les ofrecieron donde se llevó a cabo la actividad.

En la tabla 5 se presentan los resultados obtenidos en el cuestionario de satisfacción con el programa.

TABLA 5. Estadísticos descriptivos cuestionario de valoración del programa

\begin{tabular}{|c|c|c|c|}
\hline & $\mathrm{N}$ & $\mathrm{M}$ & DT \\
\hline \multicolumn{4}{|l|}{ A. Organización del curso } \\
\hline Información proporcionada & 20 & 4,55 & ,605 \\
\hline Cumplimiento de fechas y horarios & 20 & 4,55 & 605 \\
\hline Material proporcionado & 20 & 4,55 & ,605 \\
\hline \multicolumn{4}{|l|}{ B. ¿Por qué motivo decidiste asistir a estos talleres? } \\
\hline Necesario para mi formación & 20 & 4,10 & ,641 \\
\hline Poder obtener créditos de L.C. & 20 & 4,45 & ,759 \\
\hline Interés por los temas propuestos & 20 & 4,00 & 795 \\
\hline \multicolumn{4}{|l|}{ C. Expectativas } \\
\hline $\begin{array}{l}\text { Ha respondido a las expectativas que tenía de ellos } \\
\text { D. Contenidos y Metodología }\end{array}$ & 20 & 4,30 &, 571 \\
\hline Contenidos adecuados para logro de objetivos & 20 & 4,15 &, 587 \\
\hline Contenidos útiles para mi formación académica y personal & 20 & 4,50 & 607 \\
\hline Combinación adecuada entre teoría y práctica & 20 & 4,50 & ,688 \\
\hline Ejercicios y trabajos prácticos adecuados & 20 & 4,50 &, 513 \\
\hline Participación e interacción entre alumnos y con los docentes & 20 & 4,60 & ,598 \\
\hline Uso recurso didácticos y demás material utilizado & 20 & 4,30 & 657 \\
\hline \multicolumn{4}{|l|}{ E. Duración } \\
\hline Suficiente para abordar todos los contenidos & 20 & 4,15 & 671 \\
\hline Distribución horaria y espacios, adecuados & 20 & 4,40 & 681 \\
\hline El horario ha favorecido la asistencia & 20 & 4,25 & ,851 \\
\hline \multicolumn{4}{|l|}{ F. Docente } \\
\hline La forma de impartirlos ha facilitado el aprendizaje & 20 & 4,40 & ,598 \\
\hline Docentes han mostrado capacidad para solventar dudas & 20 & 4,75 & ,444 \\
\hline Docentes han generado clima que ha favorecido el aprendizaje & 20 & 4,75 & ,444 \\
\hline Instrucciones para realizar las tareas, claras & 20 & 4,65 & ,489 \\
\hline Han mostrado dominio de métodos y contenido & 20 & 4,95 & ,224 \\
\hline \multicolumn{4}{|l|}{ G. Equipamiento y medios técnicos } \\
\hline Adecuados para desarrollar los contenidos & 20 & 4,30 &, 571 \\
\hline Han sido suficientes & 20 & 4,35 &, 587 \\
\hline \multicolumn{4}{|l|}{ H. Espacios, instalaciones, mobiliario } \\
\hline Mobiliario & 20 & 4,00 & ,918 \\
\hline Temperatura & 20 & 3,75 & ,910 \\
\hline Iluminación & 20 & 4,30 & ,733 \\
\hline
\end{tabular}

I. Comentarios y sugerencias: Por último, el alumnado señaló algunos comentarios y sugerencias respecto al programa, tales como: 
"Deberían llevarse más a cabo talleres de este tipo que posibilitarán a los alumnos la dotación de las capacidades para desenvolverse mejor, por ejemplo, en exposiciones, vocabulario, toma de contacto con los demás compañeros...".

"En general, los talleres han estado bastante bien en cuanto a organización, el horario además era estupendo para nosotros y las profesoras han sido magníficas. He aprendido muchas cosas dándome cuenta de los errores que cometía. Me gustaría volver a participar en otras ediciones".

"Los talleres me han servido para darme cuenta de varios aspectos a mejorar como la ortografía, ser más crítica, etc. El resultado ha sido bastante productivo y me ha servido de mucho".

"Me parece muy interesante proponer los talleres en esta titulación porque los maestros y maestras deben estar preparados en todo lo que hemos aprendido".

\section{Conclusiones}

De acuerdo con los resultados obtenidos, se puede concluir que la propuesta llevada a cabo ha sido de interés y utilidad para los participantes, siendo generalizada la opinión de haber sido muy productiva por la variedad y riqueza temática, coincidiendo con las expectativas de los estudiantes participantes.

Parece evidente que los estudiantes noveles que inician sus estudios universitarios requieren un repertorio de estrategias que les permita desenvolverse de manera adecuada en el nuevo entorno académico, por lo que necesitan una atención específica al presentar necesidades tanto académicas como sociopersonales (García, Carpintero, Biencinto y Núñez, 2014; López-Justicia, Hernández, Jiménez, Polo y Chacón, 2008; López-Justicia, Hernández, Jiménez, Polo, Chacón y González, 2008; Rosário, Mourao, Núñez, González, Solano y Valle, 2007).

Los estudios realizados con población universitaria de nuevo ingreso coinciden con nuestra propuesta, al existir un consenso generalizado por parte de los profesionales de la educación encargados de la orientación universitaria acerca de la necesidad de poner a disposición de los estudiantes, una mayor información y asesoramiento en estrategias de hábitos de trabajo y estudio, autorregulación y habilidades personales (Arco y Fernández, 2011; García et al., 2014). De ahí que el programa de entrenamiento al que alude el presente estudio haya tratado de paliar las necesidades que presentan los estudiantes universitarios de nuevo ingreso para adecuarse lo mejor posible al nuevo escenario académico con unas demandas determinadas. La valoración que los participantes en el programa hacen del mismo coincide con los resultados obtenidos en otros estudios (Arco y Fernández, 2011; García et al., 2014; Rosário et al., 2007) en los que se puso de manifiesto que la participación de estudiantes en programas de orientación universitaria les sitúa en mejores condiciones para su éxito y permanencia en la institución (Ambroggio, 2012; GarcíaRos y Pérez-González, 2011).

Sin embargo, es necesario mencionar que acciones orientadores de esta naturaleza se enfrenta a dificultades como la participación de los estudiantes pese a ofertarla en las mejores condiciones para ellos y al ofrecimiento de ser valorada su participación con créditos de Libre Configuración, circunstancia que puede ser debida a la escasa concienciación que los estudiantes perciben sobre su propio aprendizaje. Por tanto, esta situación determina que los resultados 
obtenidos en el presente trabajo deban ser interpretados con cierta cautela, pues nos estamos refiriendo a un pequeño porcentaje de estudiantes universitarios noveles.

Finalmente, estos resultados, demuestran la necesidad y la importancia de implementar intervenciones de este tipo dado el momento de cambio que afecta a la Universidad, así, instituciones de educación superior como la European University Association (2007) promueven acciones que favorezcan una oferta real de servicios y recursos para que los estudiantes puedan adquirir mejoras en sus hábitos de trabajo y las competencias necesarias para su éxito dentro de la institución, acordes con las demandas de la universidad actual, evitando un abandono temprano.

\section{Referencias bibliográficas}

Álvarez, V. y Lázaro, A. (2002). Calidad de las Universidades y Orientación Universitaria. Málaga: Aljibe.

Álvarez, P., Pérez-Jorge D., López, D. y González, A (2014). Transición y adaptación a los estudios universitarios de los deportistas de alto nivel: la compleja relación entre aprendizaje y práctica deportiva. Revista Española de Orientación y Psicopedagogía 25(2), 74-89.

Ambroggio, G. A. (2012). Tutorías para ingresantes: experiencias en la UNC. Cuadernos de Educación. http://www.revistas.unc.edu.ar/index.php/Cuadernos/article/download/632/596

Arco, J. L. y Fernández, F. D. (2011). Eficacia de un programa de tutoría entre iguales para la mejora de los hábitos de estudio del alumnado universitario. Revista de Psicodidáctica, 16(1), 163-180.

EUROPEAN UNIVERSITY ASSOCIATION (2007). Europe's Universities beyond 2010: Diversity with a common purpose. Brussels: European University Association ASBL

Gairín, J., Muñoz, J. L., Galán-Mañas, Fernández, M. y Sanahuja, J. M. (2014). El plan de acción tutorial para estudiantes universitarios con discapacidad. Revista nacional e internacional de educación inclusiva, 7(1), 121-139.

García-Ros, R. y Pérez-González, F. (2011). Validez predictiva e incremental de las habilidades de autorregulación sobre el éxito académico en la universidad. Revista de Psicodidáctica, 16(2), 231-250 DOI: http://dx.doi.org/10.1387/RevPsicodidact.1002

García, E., Conejero, J. A. y Díez, J. L. (2014). La entrada en la Universidad: un reto para la orientación. Revista de docencia universitaria, 12(2), 255-280.

García, M., Carpintero, E., Biencinto, C. y Núñez, M. C. (2014). La evaluación del proyecto SOUestuTUtor: percepción de los mentores. Revista Complutense de Educación 25(2), 433-455.

Grau, S., Álvarez, J. D., Moncho, A., Ramos, M. C., Crespo, M. y Alonso, N. (2013). Las transiciones educativas. Necesidades de un proceso de orientación completo. Universidad de Alicante. Vicerrectorado de Estudios, Formación y Calidad. Instituto de Ciencias de la Educación.

López-Justicia, M. D., Hernández, C., Fernández, C., Polo, M. T. y Chacón, H. (2008). Características formativas y socioafectivas del alumnado de nuevo ingreso en la Facultad de Ciencias de la Educación de la Universidad de Granada. Electronic Journal of Research in Educational Psychology, 14, 95-116. 
López-Justicia, M. D., Hernández, C., Fernández, C., Polo, M. T., Chacón, H. y González, C. (2008). Lectura y escritura en alumnado universitario de educación de primer curso. Análisis de necesidades. Revista de Educación de la Universidad de Granada, 21(2), 43-60.

Michavila, F., García, J., Martínez, J., Merhi, R., Esteve, F., Martínez, A. I. (2012). Análisis de las políticas y estrategias de acogida e integración de los estudiantes de nuevo ingreso en las universidades españolas. Ministerio de Educación, Cultura y Deporte.

Olmedo, E. (2013). Enfoques de aprendizaje de los estudiantes y metodología docente: evolución hacia el nuevo sistema de formación e interacción propuesta en el EEES. RIE 31 (2), 411-429.

Pérez, J., Cañado, M. L., y Pérez, M. L. (2000). Composición escrita cuatro: cuarto curso de Educación Secundaria Obligatoria. Valladolid: La Calesa.

Rosário, P., Mourao, R., Núñez, J. C., González, J., Solano, P., y Valle, A. (2007). Eficacia de un programa instruccional para la mejora de estrategias de aprendizaje en la enseñanza superior. Psicothema, 19(3), 422-427.

Rué, J. (2014). El abandono universitario: variables, marcos de referencia y políticas de calidad. Revista de docencia universitaria. 12(2), 281-306.

Sanz, G. (2011). Prospectiva y análisis de los SIOU. Síntesis de resultados RUNAE-SIOU. X Encuentro de los Servicios de Información y Orientación Universitarios. Oviedo. Recuperado el 13 de Abril de 2015 de http://www.um.es/documents/1452701/1464662/prospectivasiou.pdf/2b49a7f3-d50f-4f52-94c1-de4e6be35518

Stufflebeam, D.L. (1996). El papel de la evaluación en la mejora escolar. El gran cuadro. En Dirección Participativa y Evaluación de Centros. II Congreso Internacional sobre Dirección de Centros. Bilbao: ICE. Universidad de Deusto.

Fecha de entrada: 9 de septiembre de 2014

Fecha de revisión: 17 de febrero de 2015

Fecha de aceptación: 20 de abril de 2015 